\title{
Lesion Sterilisation and Tissue Repair in Primary Teeth with Periapical Pathosis - A Case Series
}

\author{
${ }^{1}$ Dr.Divya.S, ${ }^{2}$ Dr.Retnakumari.N \\ Post Graduate student of Pedodontics, Govt. Dental College,Kozhikode. \\ Professor and Head of Pedodontics, Govt. Dental College, Kozhikode.
}

\begin{abstract}
Dental caries is a great challenge to the oral health of children. This is especially common among low socioeconomic status population which thrusts a great burden on community health. When proper intervention is not undertaken dental caries leads to irreversible damage of pulp. Exposure of the dental pulp to microorganisms results in the development of pulpal and periradicular pathosis. The role of these microorganisms as the main etiologic factor for pulpal pathology has been well established. One of the objectives of endodontic therapy in primary teeth with pulp necrosis and periapical pathosis is to eliminate microorganisms and their products and byproducts. Thus owing to the polymicrobial nature of root canal system infection, the use of a mixture of antibiotics to treat the diverse endodontic microflora have been proposed. In the present case series Lesion sterilization and Tissue Repair (LSTR) therapy with the combination of antibacterial drugs such as ciprofloxacin, metronidazole, and minocycline is used for disinfection of pulpal and peripical lesions of primary teeth. All the three cases showed a successful outcome at the end of three months with reduction in symptoms, increase in mineral density of bone and progressive healing of the periapical lesion. The results of these cases indicate that LSTR is an easy, simple and economical treatment option with more patient acceptance.
\end{abstract}

Keywords: LSTR, Endodontic treatment, Primary teeth, Triple antibiotic paste

\section{Introduction}

Lesion Sterilisation and Tissue Repair therapy is a novel caries, pulpal and root canal treatment system. Using an anti-bacterial drug combination, the therapy aims to eliminate causative bacteria from lesions, and after sterilization, the lesions are repaired or regenerated by the host's natural tissue recovery process. After sterilization, softened dentin will re-calcify, so both softened dentin as well as carious dentin can be intentionally left. An inflamed pulp, even with spontaneous pain, will recover after LSTR treatment. Metronidazole has a wide spectrum of bactericidal action against oral obligate anaerobes, even against isolates from infected necrotic pulps and, in fact, more than $99 \%$ of bacteria found in carious lesions and infected root dentine were not recovered in the presence of $10 \mu \mathrm{g}$ per $\mathrm{ml}$ metronidazole in in vitro experiments. However, metronidazole, even at a concentration of $100 \mu \mathrm{g}$ per ml, could not kill all the bacteria, indicating that other drugs may be needed to sterilize the infected root dentine. ${ }^{11}$ It has been reported that a mixture of antibacterial drugs, i.e., metronidazole, ciprofloxacin, and minocycline, can sterilize the root dentine. In root canal treatment, complete root canal preparation or root canal obturation is not essential due to the combination of the above mentioned antimicrobial drugs which can successfully eliminate the causative bacteria from dentin walls of the root canals. As obturation is not necessary it reduces the chair side time. It cost effective both to the dentist and to the patient as number of visits are also reduced.In the present case series we describe the use of this LSTR therapy in the healing of periapical lesions asssociated with infected primary teeth. After a follow up period of three months the patients were relieved of symptoms and the post operative radiographs showed decrease in periapical radiolucency when compared with that of preoperative radiographs. The pre operative radiographs were evaluated using the Periapical Index score (PAI score).(Table 1).

Case 1:

A six year old female child reported to the Department of Pedodontics, GDC, Calicut with the chief complaint of pain in the lower left back tooth region. The patient gives history of intermittent pain for past two weeks, with the intensity of pain increasing especially during the nights. On clinical examination deep proximal caries with pulpal exposure was seen in the lower left first primary molar. Patient had severe pain on percussion on first molar when compared to the second molar. The intraoral periapical radiograph showed periapical radiolucency corresponding to the PAI score 4 with furcation involvement and radiolucency circumscribing the distal root of the first primary molar.(Figure 1)

Endodontic treatment using the combination of antibacterial drugs namely metronidazole, minocycline, ciprofloxacin was planned for disinfection of the pulp and periapical lesion. The access opening was done in the primary first molar and the coronal pulp tissue was extripated under local anaesthesia. Then the 
triple antibiotic paste was placed in the pulp chamber over the root canal orifices and glass ionomer restoration was given as a coronal seal. Patient was reviewed after three months. Patient was asymptomatic with abscence of pain and the radiograph at the recall visit showed definite reduction in periapical radiolucency with bone formation both at the furcation area and periradicular region. (Figure 2)

\section{Case 2:}

The second case describes the endodontic treatment undergone by a six and a half year old male child reported to the Department of Pedodontics , GDC, Calicut with the chief complaint of pain and swelling in the lower left back tooth region. The patient gives history of swelling for past two days. On clinical examination, patient had dentoalveolar abcsess in relation to the left second primary molar region. The periapical radiograph showed radiolucency involving the furcation and circumscribing the mesial root of the second primary molar with PAI score corresponding to5. (Figure 3) Lesion sterilisation therapy was planned for the same tooth.

The access cavity was prepared in the lower left second primary molar. The necrotic pulp tissue was removed and the canals were irrigated with sodium hypochlorite and normal saline alternatively. Triple antibiotic mix paste was placed in the pulp chamber and the coronal seal was provided with glass ionomer cement.

After a three month follow up, the patient was asymptomatic with absence of pain and abscess in relation to left second primary molar region. The periapical radiograph showed healing of periapical lesion with increase in mineral density in bone and reduction in periapical radiolucency. (Figure 4)

\section{Case 3:}

In this case a five year old male child reported to the Department of Pedodontics , GDC, Calicut with the chief complaint of pain in the lower left back tooth region. The patient gives history of pain for past 2 weeks during night. On clinical examination left lower first primary molar had caries with pulpal exposure. There was dentoalveolar abscess in relation to the same tooth The radiograph showed mild furcation involvement. (Figure 5) At this visit the access cavity was prepared and the pulp was extripated. The root canals were irrigated with sodium hyppchlorite and normal saline , triple antibiotic paste was placed in the pulp chamber and sealed with glass ionomer cement. Patient was completely relieved of symptoms and abscess had subsided by the end of three months. The periapical radiolucency had significantly reduced . (Figure 6)

\section{Discussion:}

The teeth with infected root canals, particularly those in which infection has reached the periradicular tissues are a common problem in primary dentition. Early loss of primary teeth can cause number of problems, such as - ectopic eruption, disturbance of eruption sequence, drifting of erupted teeth, space loss for the successor permanent teeth, development of aberrant habits such as tongue thrusting, alterations in speech, and impairment of function. Thus it is important that primary dentition should be maintained in the dental arch, provided it can be restored to function and remain free from the disease.

Endodontic therapy plays an important role in removing bacteria, their by-products and their substrates, by disrupting and destroying the microbial ecosystem through chemical and mechanical methods. Different drugs and medicaments have also been suggested to accompany these techniques with varying success rate. Metronidazole has a wide spectrum of bactericidal action against oral obligate anaerobes. It has been found that obligate anaerobes form majority of isolates from carious lesions, infected root dentin, and from nonexposed pulp tissue. However, metronidazole even at higher concentrations could not eradicate all the bacteria from carious lesions, indicating the necessity of some additional drugs to sterilize these lesions. ${ }^{2}$ Tetracycline is known to enhance the growth of host cells on dentin, not by antimicrobial action, but via the exposure of embedded collagen fibers or growth factors. Triple antibiotic paste, on the other hand, contains both bactericidal (metronidazole, ciprofloxacin) and bacteriostatic (minocycline) components. ${ }^{3}$ Thus, it was seen that a mixture of antibacterial drugs, i.e. ciprofloxacin, etronidazole, and minocycline can sterilize carious lesions, necrotic pulps, and infected root dentine of deciduous teeth. ${ }^{2}$ The combination of drugs has been shown to penetrate efficiently through dentine from the prepared root canals especially from the ultrasonically irrigated root canals. The commercially available drugs are powdered and mixed in a ratio of 1:3:3 (3 Mix) and mixed either with macrogol-propylene glycol (3 Mix-MP) or a canal sealer (3 Mix-sealer). A 1:1:1 ratio of the drug combination has also been used. Although the volume of the drugs applied in this therapy is small, care should be taken to check if the patients are sensitive to chemicals or antibiotics. A disadvantage of the triple antibiotic paste is tooth discoloration induced by minocycline. Cefaclor and fosfomycin are proposed as possible alternatives for minocycline, in terms of their antibiotic effectiveness.$^{3}$

Windley et al $(2005)^{4}$ in the animal study showed that a statistically significant reduction in bacteria, cultured from infected immature dog teeth, was found following the irrigation and antibiotic paste protocol used 
in this study. Of the 30 samples that cultured bacteria before treatment, $90 \%$ remained positive following irrigation with $10 \mathrm{ml}$ of $1.25 \% \mathrm{NaOCl}$. However, this dropped to $30 \%$ following the application of the triple antibiotic paste for $2 \mathrm{wk}$.

Akgun et al (2009) $)^{5}$ used triple antibiotic paste as a disinfectant for a traumatized immature tooth with a periapical lesion and found that after 4 months, the patient had no symptoms, and a radiograph showed the radiolucency had completely resolved.

Raison Bose compared TAP, calcium hydroxide, and formocresol as intracanal medicaments in nonvital young permanent tooth. The triple antibiotic group showed the highest percentage increase in the dentin wall thickness compared with the other two groups. TAP can help promote functional development of the pulpdentin complex. Reynolds $e$ e al. achieved revascularization of a necrotic bicuspid using TAP.TAP contains both bactericidal metronidazole, ciprofloxacin) and bacteriostatic (minocycline) agents to allow for successful revascularization. ${ }^{6}$

Tauksheje et al(2004) $)^{7}$ evaluated the effectiveness of LSTR in primary teeth. They found that in 87 cases after a follow up period of 680 days found that infected primary teeth were relieved of symptoms such as abscess, gingival fistulae and pain and the teeth remained asymptomatic until their exfoliation.

Prabakar et al $(2008)^{2}$ evaluated clinical and radiographic success of endodontic treatment of infected primary teeth using combination of ciprofloxacin, metronidazole, and minocycline.

They found that endodontic treatment using antibacterial mix (a combination of ciprofloxacin, metronidazole, and minocycline mixed with propylene glycol and macrogol) in primary teeth has shown good clinical and radiographic success. They also found that all the treated cases were clinically and radiographically successful with removal of necrotic coronal and accessible radicular pulp tissue when compared with only coronal pulp removal.

Manisha Agaerwal et al $(2011)^{8}$ assessed the efficacy of LSTR and pulpotomy against the conventional pulpectomy treatment. They concluded that LSTR and pulpotomy were effective but long term follow up is needed for LSTR to consider its efficacy.

Jaya et al (2012) $)^{9}$ evaluated and compared the clinical and radiographic effectiveness of Ciprofloxacin, Minocycline, Metronidazole combination with Ciprofloxacin, Minocycline and Tinidazole combination when used for Lesion Sterilization and Tissue Repair in primary teeth . they observed no statistically significant difference between both the groups and thus a combination of Ciprofloxacin, Minocycline and Tinidazole antibacterial drugs can be used on teeth pulpally involved with physiologic root resorption.

In the present case series a combination of metronidazole, ciprofloxacin and minocycline was used. Commercially available tablets Ciprofloxacin 500mg, Metronidazole 200mg, Minocycline 100mg were purchased and powdered with mortar and pestle. A ratio of 1:1:1 was used to prepare the antibiotic mixture. ${ }^{10}$ The powder was mixed with commercially available ciprofloxacin ointment at the time of treatment and placed in the pulp chamber.

It was observed that in all the three cases symptoms such as pain and swelling resolved and there was a reduction in the periapical radiolucency as indicated by the PAI score ${ }^{11}$ within three months. This shows that LSTR is an effective treatment in the management of infected primary teeth. In most cases, the treatment can be completed in one visit and patient acceptance is more. As the primary teeth is preserved more complicating procedures such as extraction followed by fabrication of space maintainer appliances are eliminitated. Thus this procedure is both less time consuming and cost effective.

\section{Conclusion:}

The Lesion sterislisation ant tissue repair therapy is simple, painless, time-saving, and with less of a burden to patients physically and mentally. Thus, patient compliance and cooperation of patients is predicable,which is of great concern in the management of Pediatric patients. This procedure might disinfect the severely infected deciduous teeth and allow it to function as a space maintainer until the eruption of its permanent successor. This new concept should be considered especially in cases where prognosis is poor with the conventional pulpectomy procedure in primary teeth with furcation involvement and severe bone loss . 
Figure 1: Case 1 - Preoperative radiograph

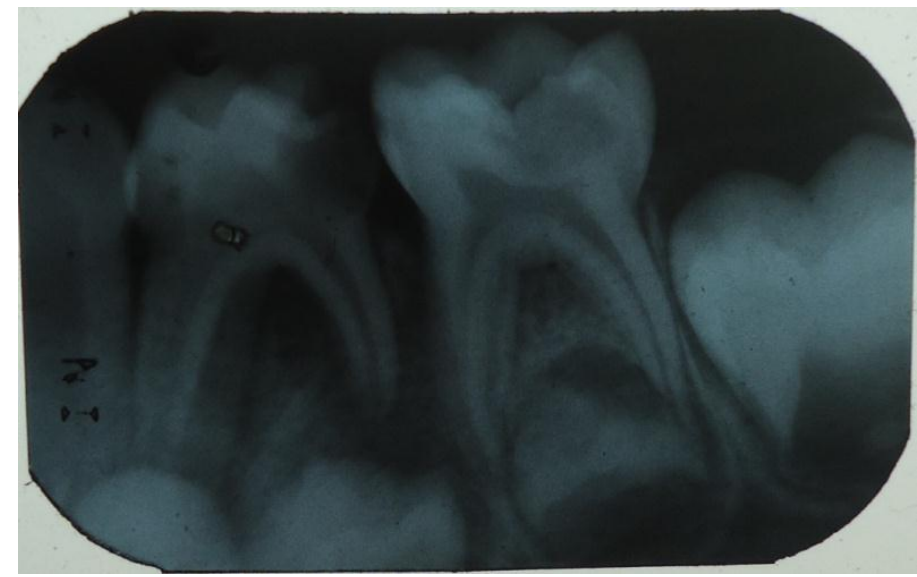

Figure 2: Case 1 Postoperative - 3month follow up

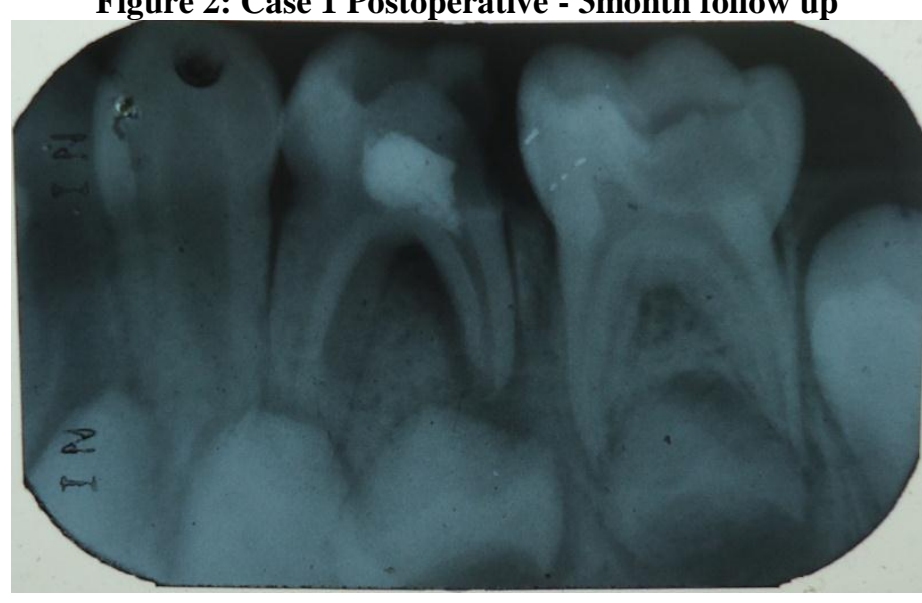

Figure 3: Case 2 Preoperative

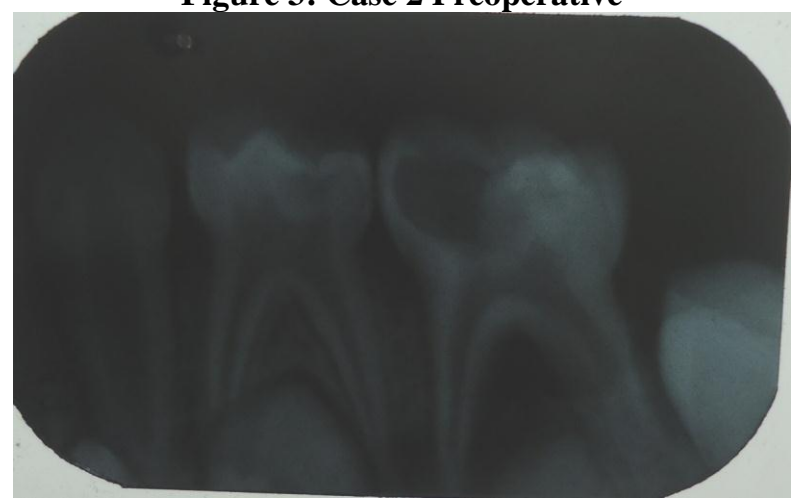

Figure 4: Case 2 Postoperative - 3month follow up

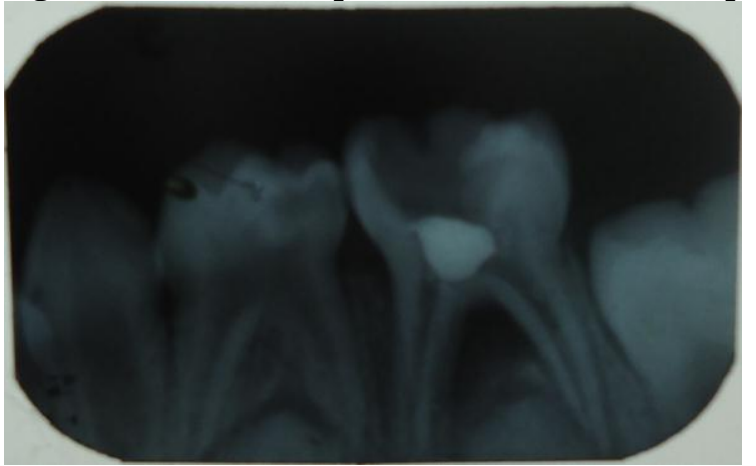



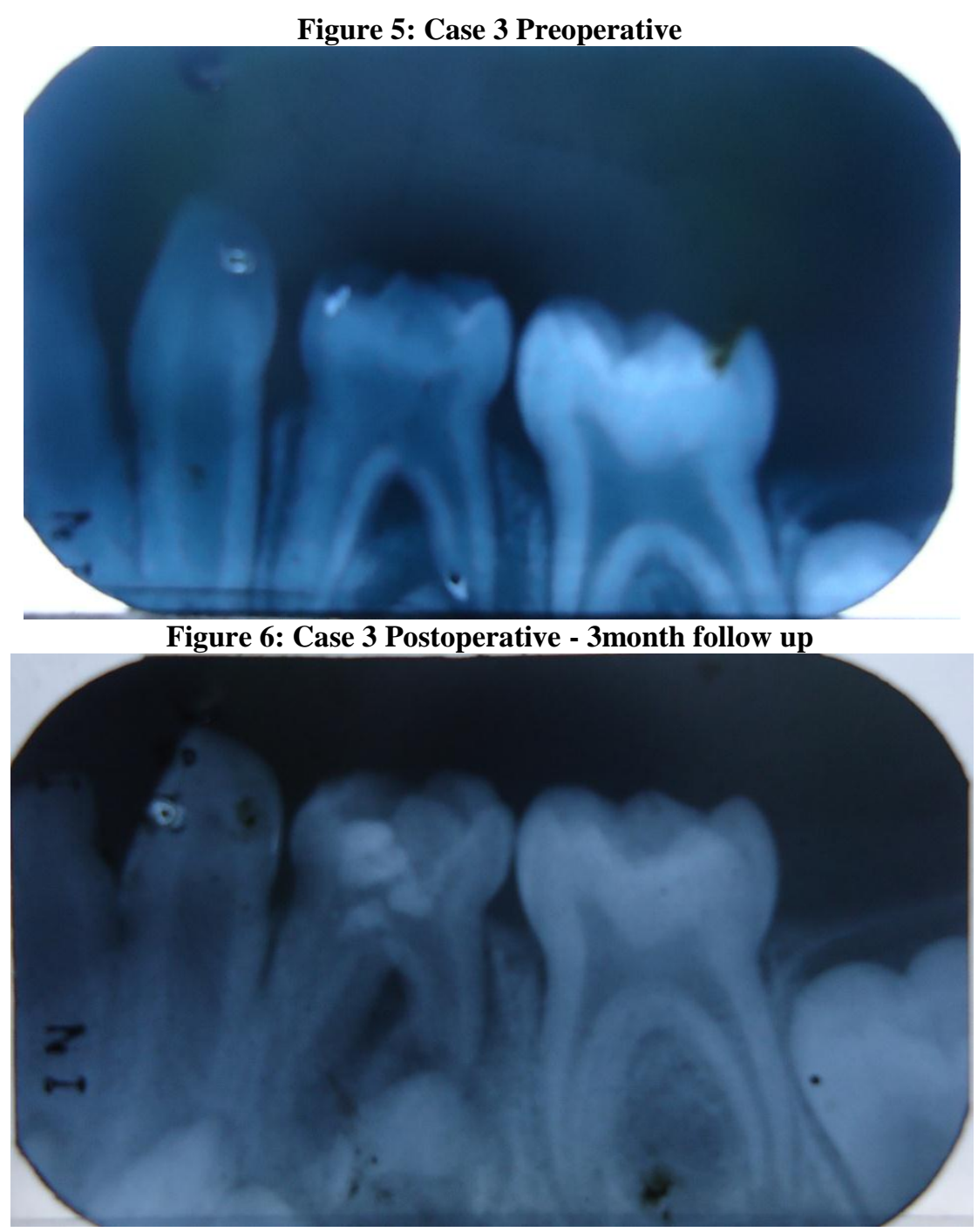

Table 1

PAI index

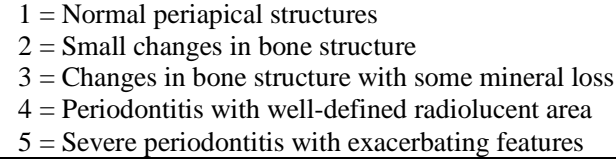

\section{References:}

Sonali Taneja, Manju Kumari, and Hari Parkash, Nonsurgical healing of large periradicular lesions using a triple antibiotic paste: A case series. Contemp Clin Dent. 2010; 1(1): 31-35.

[2]. Prabhakar A. R, Sridevi E, Raju O. S, Satish V. Endodontic treatment of primary teeth using combination of antibnacterial drugs: An in vivo study.J Indian Soc Pedod Prevent Dent - Supplement 2008

[3]. Marina Fernandes and Ida de Ataide, Nonsurgical management of periapical lesions. J Conserv Dent. 2010 Oct-Dec; 13(4): 240245 .

[4]. Windley et al, Triple Antibiotic Paste and Bacterial Reduction. JOE - Volume 31, Number 6, June 2005.

[5]. Akgun et al,Use of triple antibiotic paste as a disinfectant for a traumatized

[6]. immature tooth with a periapical lesion: A case reportOral Surg Oral Med Oral Pathol Oral Radiol Endod 2009;108:e62-e65

[7]. Vijayaraghavan et al.Triple antibiotic paste in root canal therapy, J Pharm Bioallied Sci. 2012 August; 4(Suppl 2): S230-S233.

[8]. Takushige et al, Endodontic treatment of primary teeth using a combination of antibacterial drugsInternational Endodontic Journal $.2004,37,132-138$

[9]. Agarwal et al, A comparative evaluation of non instrumentation endodontic techniques with conventional ZOE pulpectomy, World Journal of Dentistry, 2011;2(3):187-192.

[10]. Jaya et al, In vivo evaluation of lesion sterilization and tissue repair in primary teeth pulp therapy using two antibiotic drug combinations. J Clin Pediatr Dent. 2012 Winter;37(2):189-91.

[11]. Varalakshmi "3Mix- MP in Endodontics - An overview" IOSR Journal of Dental and Medical Sciences (JDMS) ,2012,3(1) 36-45

[12]. Vytaute Peciuliene, Apical periodontitis in root filled teeth associated with the quality of root fillings Stomatologija, Baltic Dental and Maxillofacial Journal. 2006;8:122-6. 